\title{
ESTUDIO DE ERGONOMÍA ORGANIZACIONAL Y PARTICIPATIVA EN UN CENTRO DE SALUD EN CHILE
}

\section{ORGANIZATIONAL AND PARTICIPATORY ERGONOMIC STUDY IN A PRIMARY CARE HEALTH CENTER IN CHILE}

\author{
Javier Alonso Freire-Herrera* \\ Violeta Lorena Mera-Salinas ${ }^{* *}$
}

\begin{abstract}
Resumen: La mayoría de los estudios ergonómicos se han concentrado en el personal clínico de salud y pocas investigaciones han explorado las condiciones en que laboran las trabajadoras administrativas que se desempeñan en centros de salud. El objetivo de este estudio fue identificar los riesgos organizacionales y ergonómicos en un centro de salud de atención primaria para recomendar mejoras en el desarrollo de las actividades de las trabajadoras administrativas. Se realizaron entrevistas semi estructuradas, además de observación directa de las actividades y condiciones de trabajo de las funcionarias. Se detectó que uno de los principales problemas referidos por las trabajadoras y corroborados por la observación, estaban asociados a restricciones de espacio que repercutían en molestias corporales. También se identificaron falencias en el ambiente físico como el calor y el frío por mal estado de la calefacción central. Por otra parte, se identificaron problemas con la seguridad y el bienestar de las trabajadoras derivados de situaciones de agresividad y malos tratos por parte de los usuarios. Estos datos proporcionan una visión fundamental de la necesidad de intervenir en mejorar las condiciones laborales de las trabajadoras administrativas del área de la salud. Estos hallazgos tienen posibles implicaciones para la salud y seguridad de las trabajadoras administrativas. En conjunto, estos resultados ofrecen una visión global de la importancia de incluir un programa participativo en el abordaje de los problemas ergonómicos. Es necesario seguir trabajando para abordar con una mirada multidisciplinaria la solución a problemas complejos del área de la salud.
\end{abstract}

Palabras clave: Ergonomía participativa, atención primaria, centro de salud, Latinoamérica.

\begin{abstract}
Most of these ergonomic studies have focused on clinical health personnel. Few studies have explored the working conditions of administrative workers in health centers. The objective of this study was to identify organizational and ergonomic risks in a primary care health center to recommend improvements in the performance of administrative workers' activities. To further elucidate the characteristics of the work, semi-structured interviews were conducted with the administrative workers and direct observation of the activities and working conditions of the employees. It was identified that one of the main problems reported by the workers and corroborated by observation were problems associated with space restrictions that resulted in bodily discomfort. We also identified shortcomings in the physical environment, such as heat and cold due to central heating problems. On the other hand, serious issues were identified regarding the safety and well-being of the workers due to users' aggressiveness and mistreatment. These data provide fundamental insight into the need for
\end{abstract}

\footnotetext{
"Departamento de Ergonomía, Universidad de Concepción. Concepción, Chile. Correo electrónico: javierfreire@udec.cl. Orcid: https://orcid.org/oooo-0002-9757-5577. Autor de correspondencia.

** Dirección Administración Municipal de Salud. Hualpén, Chile. Correo electrónico: vmera83@gmail.com. Orcid: https://orcid.org/oooo-0001-7021-917X
} 
intervention to improve the working conditions of female health care administrative workers. These findings have potential implications for the health and safety of female administrative workers. Taken together, these results provide a comprehensive overview of the importance of including a participatory program in addressing ergonomic problems. Further work is needed to take a multidisciplinary approach to solve complex problems.

Keywords: Participatory ergonomics, primary care, health center, Latin America.

Recepción: 19.05.2021 / Revisión: 25.05.2021 / Aceptación: 29.07.2021

\section{Introducción}

El sistema de salud en Chile se caracteriza por presentar un carácter mixto entre atención privada y pública. A su vez, el sistema público es organizado por el Sistema Nacional de Servicios de Salud (SNSS) y por el sistema de atención primaria (APS) administrados por las municipalidades (Goldstein, 2018).

El objetivo de la Atención Primaria es otorgar una atención equitativa y de calidad, centrada en las personas y sus familias, enfocada en lo preventivo y promocional, es decir, anticipándose a la enfermedad, bajo el Modelo de Salud Integral con Enfoque Familiar y Comunitario, que es un modelo de atención centrado en las personas, sus familias y la comunidad, con un equipo de salud de cabecera que brinda atención continua a lo largo de todo el ciclo de vida (Servicio de Salud Metropolitano Norte, s.f.).

En estos centros de salud no sólo trabajan funcionarios de salud, sino que además trabajadores administrativos. Las actividades de las trabajadoras administrativas corresponden en general a la admisión o dación de horas a usuarios para consultas y controles de los profesionales. Además, realizan la preparación de documentación necesaria para atención de usuario, con su ficha clínica ordenada y en condiciones de ser utilizada.

Existen diversos estudios que concluyen sobre las deficientes condiciones laborales de los trabajadores del área de la salud en temas relacionados como la violencia verbal y física, robos, deficientes condiciones ambientales entre otros aspectos. Cuando la demanda de las actividades es permanente y no se encuentra balanceadas con las capacidades y herramientas laborales, se produce una sobrecarga que deriva en aumento de licencias médicas y por consecuente ausentismo. Existe una relación entre la calidad de vida del personal de salud y el ausentismo. El ausentismo laboral es un claro síntoma de problemas en la gestión de los recursos humanos dentro de una organización (Vidal-Gamboa et al., 2017).

Las tasas de ausentismo laboral más altas se encontraron en el sector hospitalario: la tasa de incapacidad fue de 14,3 días por trabajador, la tasa de frecuencia de 1,3 licencia médica curativa anual y la tasa de severidad de 10,6 días en cada licencia médica curativa (Mesa \& Kaempffer, 2004).

Otro punto importante a destacar, en Chile el ausentismo laboral por causa médica es la primera de las tres causas que generan mayor gasto público en salud (Dirección de Presupuestos [DIRPRES], 2020). 
Las características ergonómicas y organizacionales están bien descritas en la literatura en relación con los profesionales de la salud. No obstante, no se dispone de suficiente información en relación con las demandas de las tareas del personal administrativo en los centros de salud. El objetivo central de este estudio fue identificar problemas organizacionales y ergonómicos en un centro de salud de atención primaria para recomendar mejoras en el desarrollo de las actividades.

\section{Materiales y métodos}

Se realizó una identificación de problemas ergonómicos de los puestos de trabajo administrativos de un centro de salud en la región del Biobío. El estudio fue desarrollado en base al modelo de ergonomía participativa, que involucra cinco etapas en su aplicación las cuales son; la motivación de participación, participación en el proceso, competencia, confianza y resultados (Haines \& Wilson, 1998).

Se realizaron reuniones de motivación a nivel de la administración de salud, como también con las trabajadoras administrativas en el comienzo del proyecto. Posteriormente se consideró la participación de todas las trabajadoras administrativas en las entrevistas e identificación de riesgos ergonómicos en sus puestos de trabajo. Las recomendaciones finales fueron consensuadas entre los investigadores del proyecto, la administración y las trabajadoras.

Para indagar sobre las variables organizacionales y ergonómicas, se aplicaron entrevistas semiestructuradas, debido a que es uno de los instrumentos cuyo propósito es recabar datos y permite obtener información más profunda, detallada, que incluso el entrevistado y entrevistador no tenían identificada, ya que se adapta al contexto (Díaz-Bravo et al., 2013).

Se consideraron variables como espacio de trabajo, ambiente térmico, iluminación, violencia entre otras variables, que fueron incorporadas en las entrevistas e inspección en terreno. Para llevar a cabo las evaluaciones se necesitó cámaras fotográficas, grabadoras, materiales de oficina y computadora.

\section{Resultados y discusión}

Los resultados obtenidos en este estudio evidencian diversos aspectos que son abordados en distintas áreas temáticas.

\section{Restricciones de espacio}

Se identificaron severas restricciones de espacio en algunos puestos de trabajo. En la figura 1, se observa a la trabajadora encargada de recepción de los reclamos y sugerencias de los usuarios. El espacio de trabajo no supera los $4 \mathrm{~m} 2$ y su jornada es de 8 horas por día. Se observaron posturas sostenidas por largo tiempo y restringidas producto del poco espacio 
disponible para la ubicación de elementos como la pantalla, teclado, teléfono entre otros elementos necesarios. Debido a la restricción de espacio la pantalla del computador la debe ubicar en el mesón destinado para la atención de usuarios. El asiento utilizado carece de elementos como apoyabrazos lo que se traduce en una constante contracción de musculatura de la extremidad superior. Por otra parte, en la parte inferior del mesón de trabajo se identificó el acopio de elementos como carpetas, cajas de insumos entre otros objetos.

Figura 1. Restricciones de espacio en funcionaria.
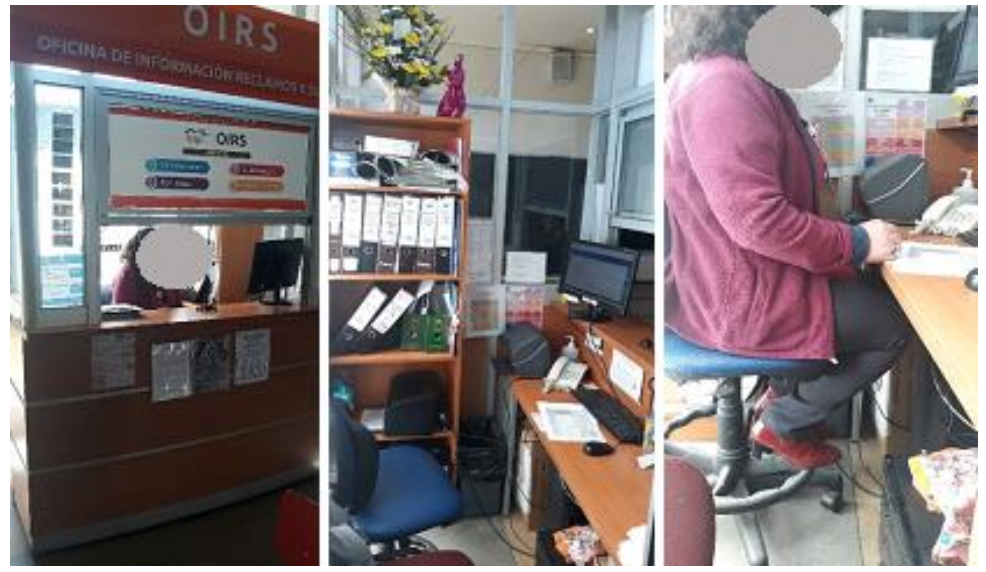

El acopio de cajas y elementos bajo los escritorios de trabajo fue observado en otros puestos de trabajo (figura 2), ya que debido a la ausencia de una bodega cercana a los puestos de trabajo se opta por guardar los elementos en los propios puestos de trabajo. Una de las consecuencias de esta práctica, es la limitación de la movilidad de las extremidades inferiores restringiendo el cambio postural.

Figura 2. Acopio de cajas.

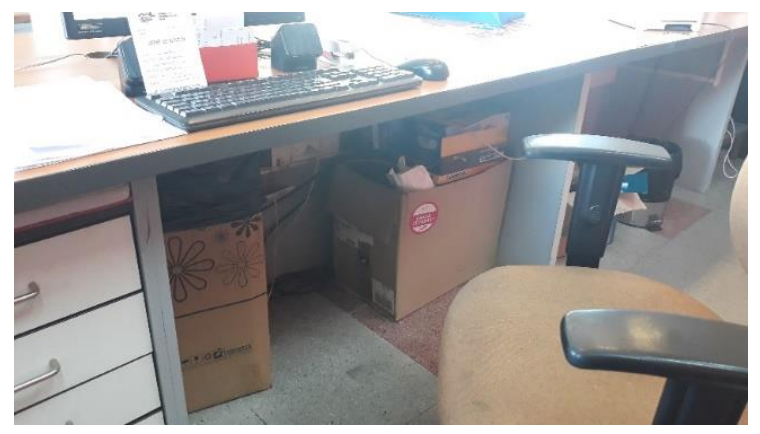

Otro aspecto importante en la limitación del espacio es la utilización de cardex (figura 3) para guardar las fichas clínicas de papel que ya se encontrarían en desuso producto de la implementación de la ficha electrónica de los pacientes. De acuerdo a lo comentado por las funcionarias, se ha solicitado en diversas oportunidades el retiro de estos elementos ya que utilizan mucho espacio y causan problemas de alergia por los ácaros del polvo de las fichas en papel. Lamentablemente no se ha concretado la solicitud por problemas de financiamiento en la transformación del espacio. 
Figura 3. Cardex de fichas clínicas.

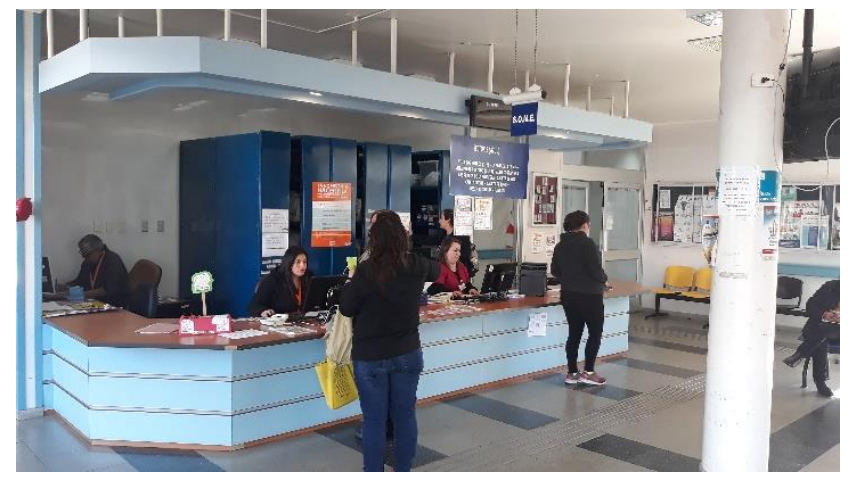

El espacio efectivo de trabajo es un elemento esencial del bienestar laboral. Según el arquitecto Greg Dunlop existe menos espacio disponible para trabajar, ya que sólo $25 \%$ del espacio de oficina está destinado a las labores de los diferentes empleos. El resto es ocupado por papelería, archivadores, computadoras, archivos y demás equipos periféricos. Esta misma situación fue observada en la evaluación de los distintos puestos de trabajo administrativo de este centro de salud.

\section{Sistema de calefacción deficiente}

Por otra parte, se identificó un deficiente sistema de calefacción debido a una falla en la caldera, lo que se evidencia en la figura 4. Al ser consultado por su reparación, la respuesta fue la limitación de financiamiento por el alto costo que implica. Esto repercute en la sensación de frío no sólo de los funcionarios, sino que además de los pacientes. Para poder mitigar el efecto del ambiente térmico en invierno, se utilizan calefactores eléctricos. No obstante, el sistema eléctrico no tiene las mejores condiciones de mantención.

Figura 4. Radiador sin funcionamiento.

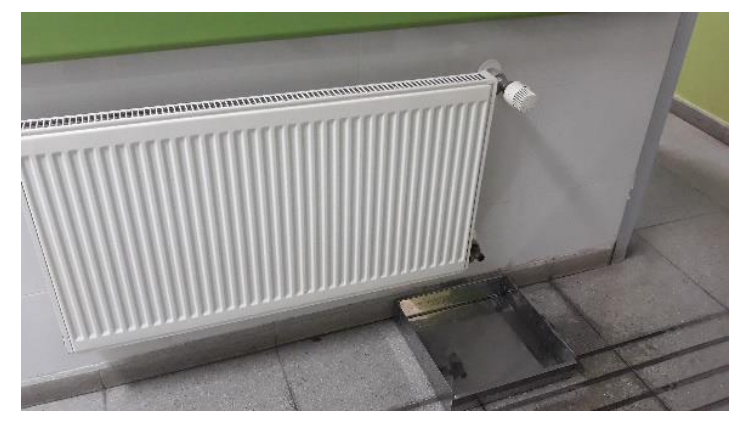

\section{Sistema eléctrico deficiente}

Se identificaron problemas con las conexiones eléctricas que se encuentran en deficientes condiciones pudiendo ser un potencial riesgo de accidente eléctrico o foco de un incendio. Esta característica fue observada en la mayoría de los puestos de trabajo como se observa en la figura 5 . 
Figura 5. Enchufes en mal estado.
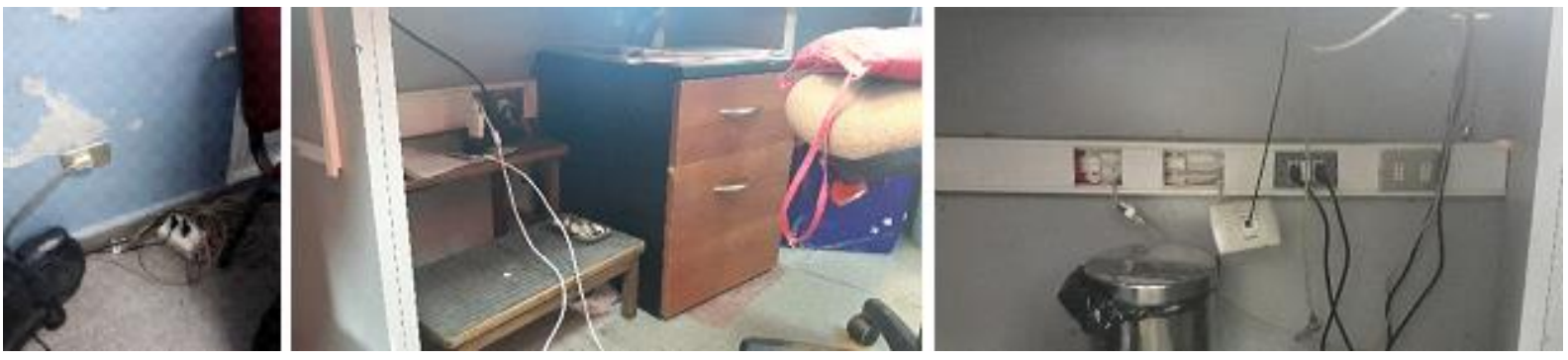

Agresividad y robos de usuarios

Las trabajadoras reportaron situaciones de violencia verbal y física por parte de usuarios, debido a situaciones como falta de horarios de atención, cancelación de horas con profesionales de la salud por retraso, estado etílico de pacientes, entre otras razones. En la figura 6 , se puede apreciar que las trabajadoras administrativas tienen directa relación con la atención de los usuarios sin ninguna barrera de protección frente a hechos de violencia por parte de los usuarios. Desde la dirección de salud municipal están consiente de esta problemática y están incorporando un sistema de atención dentro de un espacio cerrado que mantenga la separación con los usuarios como se observa en la figura 7. No todos los puestos de trabajo están cerrados por falta de recursos en la habitación de los módulos.

Figura 6. Módulos de atención abiertos.

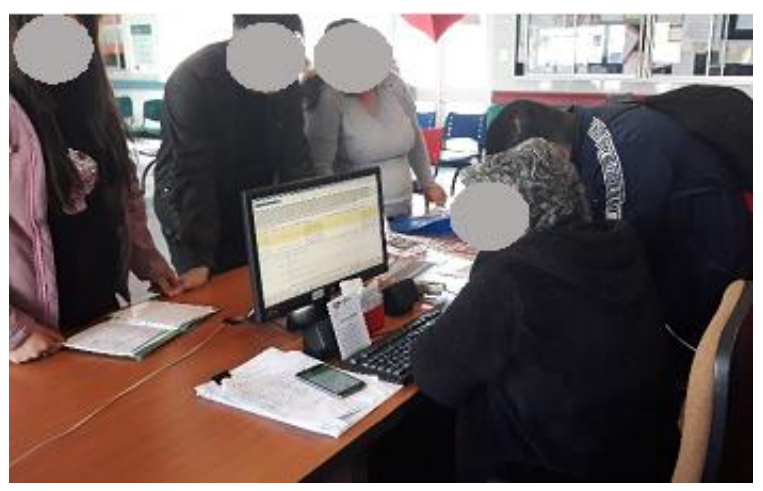

Figura 7. Módulos de atención cerrados.

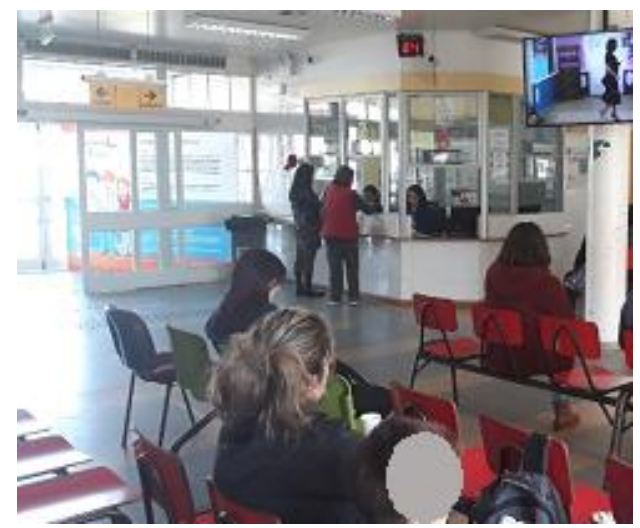


Por otra parte, se repiten frecuentemente robos de artículos personales de las trabajadoras como de artículos de oficina o insumos médicos. Se han adoptado diversas medidas como cámaras de vigilancias, guardias de seguridad, protocolo de actuación frente a robos como retirar elementos que se puedan robar de los puestos de trabajo.

De acuerdo con diversos estudios, se ha considerado que el trabajo en el área de la salud es uno de lo más expuestos a la violencia por parte de los usuarios, lo que expone a los trabajadores a una constante demanda laboral produciendo alteraciones de la salud mental (Itzhaki et al., 2015).

Existe una asociación entre la exposición a factores de riesgo psicosociales y la exposición a violencia laboral y el distrés elevado, medidos en tres centros de salud en Chile (Palma-Contreras \& Ansoleaga, 2020).

De acuerdo con el informe anual de estadísticas sobre seguridad y salud en el trabajo, las enfermedades mentales han aumentado en los últimos años, representando el $38 \%$ de las enfermedades profesionales en Chile (Superintendencia de Seguridad Social [SUSESO], 2020). Otro antecedente relevante es que en el $73 \%$ de las mujeres las enfermedades profesionales están asociadas a enfermedades mentales.

\section{Actividades de riesgo físico y accidente}

En el puesto de trabajo de la encargada de bodega, se observaron numerosas acciones de riesgo al momento de manipular los distintos elementos de aseo y de oficina. Como se comentó anteriormente existe un problema de espacio físico, por lo que se utilizan hasta los últimos rincones para guardar objetos. Por lo anterior, la trabajadora debe subirse sobre un mueble para lograr alcanzar los elementos superiores, y además se observó que en una zona de tránsito de funcionarios existe un mal diseño de una ventana, la cual al estar abierta limita el libre tránsito, como se observa en la figura 8. Se reportaron un par de accidentes debido a esta situación.

Figura 8. Situaciones con potencial riesgo de accidentes.
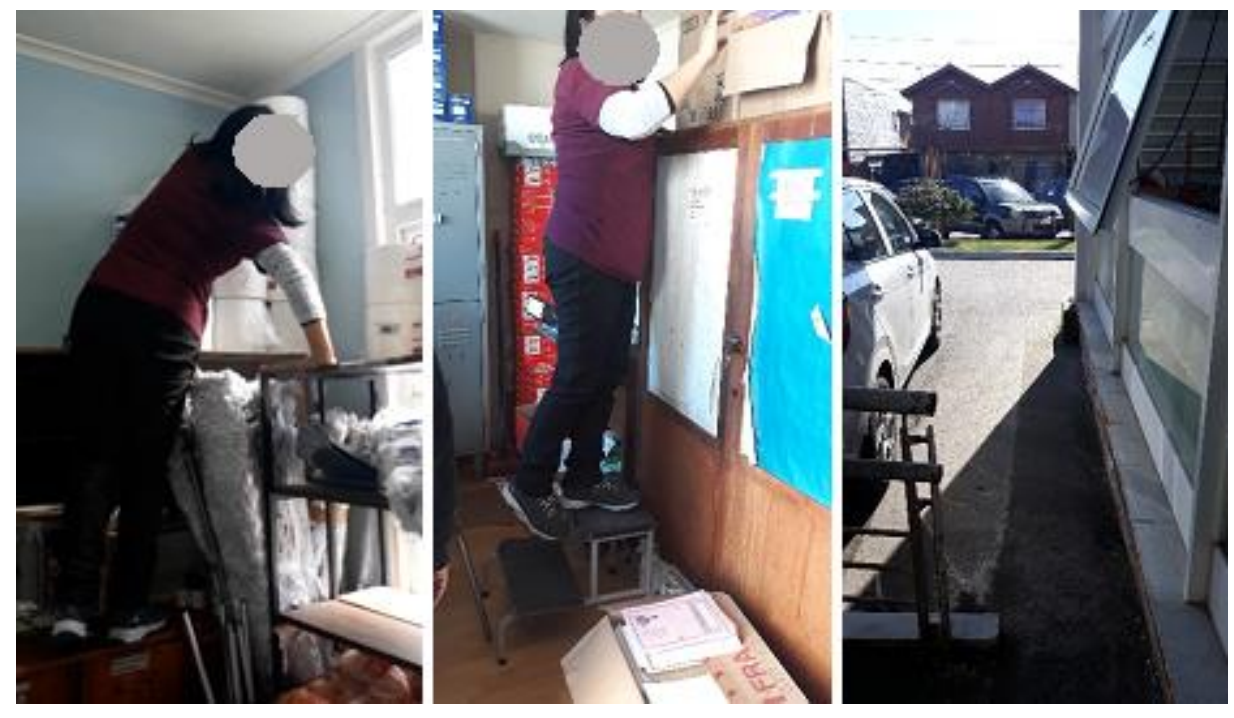


\section{Conclusiones}

Una de las conclusiones más significativas de este primer estudio, es la necesidad de implementar mejoras en la organización y en temas relacionados a ergonomía que no han sido abordados en etapas de diseño ni de corrección por temas presupuestarios.

Este estudio ha demostrado que existen variables como las características del mobiliario, espacios reducidos, ambiente físico y violencia que afectan el bienestar laboral de las trabajadoras administrativas.

Se propone que los espacios de trabajo puedan estar diseñados y consideren las características antropométricas de las trabajadoras con el objetivo de lograr una correcta organización.

En base a las condiciones identificadas se hace necesario continuar con un modelo participativo en la detección de otras variables que afectan la salud y seguridad de las trabajadoras.

En relación con situaciones de agresividad y malos tratos por parte de los usuarios, se recomienda implementar un protocolo de actuación frente a hechos de violencia. Este protocolo debe contemplar una correcta y oportuna etapa de identificación de estas situaciones. Por otra parte, se deben disponer de un canal rápido que permita ir en ayuda de la funcionaria que está siendo afectada. Aumentar el número de guardias en los centros de salud y contar con un canal directo a la policía más cercana.

Finalmente, se propone seguir avanzando en la profundidad del estudio con el objetivo de generar un manual de buenas prácticas ergonómicas en los centros de salud de las distintas regiones del país. Por otra parte, se propone crear un estándar con diversas características de las trabajadoras y de las actividades que se desarrollan que sirva de modelo cuando se diseñen y construyan nuevos establecimientos de salud. Se espera que el estudio pueda continuar en este centro, como así en otros centros de salud donde la realidad frente a situaciones como esta puede ser igual o con mayores dificultades. 


\section{Referencias}

Díaz-Bravo, L., Torruco-García, U., Martínez-Hernández, M., \& Varela-Ruiz, M. (2013). La entrevista, recurso flexible y dinámico. Investigación en Educación Médica, 2(7), 162-167. https://doi.org/10.1016/s2007-5057(13)72706-6

Dirección de Presupuestos (2020). Anuario estadístico del empleo público en el Gobierno Central 2011-2019. http://www.dipres.gob.cl/598/w3-propertyvalue-15676.html

Goldstein, E. (2018). El sistema de salud en Chile y la atención primaria de salud municipal. https://obtienearchivo.bcn.cl/obtienearchivo?id=repositorio/10221/26811/2/BCN Goberna nza salud y demunicip para reposit final.pdf

Haines, H. M., \& Wilson, J. R. (1998). Development of a framework for participatory ergonomics. Health and Safety Executive. http://www.opengrey.eu/item/display/10068/374914

Itzhaki, M., Peles-Bortz, A., Kostistky, H., Barnoy, D., Filshtinsky, V., \& Bluvstein, I. (2015). Exposure of mental health nurses to violence associated with job stress, life satisfaction, staff resilience, and post-traumatic growth. International Journal of Mental Health Nursing, 24(5), 403-412. https://doi.org/10.1111/inm.12151

Mesa, M, F. R., \& Kaempffer, R. A. M. (2004). 30 años de estudio sobre ausentismo laboral en Chile: Una perspectiva por tipos de empresas. Revista Médica de Chile, 132(9), 1100-1108. https://doi.org/10.4067/s0034-98872004000900012

Palma-Contreras, A., \& Ansoleaga, E. (2020). Associations between psychosocial risk factors, organizational dimensions, and mental health problems related to workplace violence among workers of three chilean hospitals of high complexity. Cadernos de Saude Publica, 36(3), eooo84219. https://doi.org/10.1590/0102-311x00084219

Servicio de Salud Metropolitano Norte (s.f.). Atención primaria. Consultado el 26 de agosto de 2020. https://www.ssmn.cl/atencion_primaria.ajax.php

Superintendencia de Seguridad Social (2020). Informe anual: Estadísticas sobre seguridad y salud en el trabajo 2019. https://www.suseso.cl/607/w3-article-595996.html

Vidal-Gamboa, C., Palavecino-Sáez, I., Rivera, P. M., Huerta, C. T., \& Hoffmeister-Arce, L. (2017). Calidad de vida del personal de salud y su relación con el ausentismo. Ciencia y Trabajo, 19(60), 188-193. https://doi.org/10.4067/So718-24492017000300188 